\title{
AdENOCARCINOMA PRIMÁRIO DO DUODENO
}

\author{
Pablo Rodrigo de Siqueira, Sidney Roberto Nadal, francisco César Martins Rodrigues, Carlos Alberto Malheiros* \\ Trabalho realizado na Área V - Grupo de Estômago e Duodeno do Departamento de \\ Cirurgia da Faculdade de Ciências Médicas da Santa Casa de São Paulo, S. Paulo, SP.
}

RESUMO - OBjetivo. Revisar a literatura especializada a respeito dos adenocarcinomas duodenais primários e comparar os dados com os aspectos observados nesta casuística.

Mérodos. Seis doentes com esta neoplasia, excluídas as lesóes periampulares, foram operados na Área de Estômago e Duodeno do Departamento de Cirurgia da Santa Casa de Säo Paulo, entre 1991 e 1999. Houve predominância do tumor no sexo masculino e na $6^{a}$ década de vida. Os sinais e sintomas mais freqüentes foram o emagrecimento $(83,3 \%)$ e a obstrução intestinal crônica (66,6\%), com média de duraçäo de seis meses. A gastroduodenoscopia fez o diagnóstico, posteriormente confirmado pela histologia. Utilizamos a tomografia computadorizada e a ultrasonografia para determinar 0 estádio da doença. Em três pacientes com lesão restrita à parede do órgão, praticou-se a ressecção duodenal segmentar curativa, e nos outros três, a derivação duodenal paliativa, pois 0 tumor infiltrava órgãos adjacentes.
Resultados. 0 tempo de internação pós-operatória variou de 5 a 9 dias, com média de 7,3 dias. A mortalidade operatória foi $16,6 \%$ (um doente). Não indicamos tratamento complementar com quimio ou radioterapia. 0 acompanhamento ambulatorial vem ocorrendo em períodos que variam de 15 a 20 meses. Os três pacientes submetidos à ressecção cirúrgica curativa estäo sem evidências de recidiva até $015^{\circ}, 16^{\circ}$ e $18^{\circ}$ mês de pós-operatório. Houve seguimento de um e três meses nos dois pacientes submetidos a cirurgias paliativas, depois do que se perderam.

Conclusão. 0 pequeno número de casos relatados não permitiu conclusōes mais consistentes a respeito dos adenocarcinomas duodenais.

UNITERMOS: Adenocarcinoma primário. Duodeno.

\section{INTRODUÇÃO}

O carcinoma primário de duodeno, excluindo os da região da ampola de Vater, é extremamente raro. Representa cerca de $0,35 \%$ dos tumores malignos do trato gastrointestinal e $25 \%$ a $45 \%$ dos neoplasmas malignos do intestino delgado $0^{1-4}$. O duodeno compreende apenas $8 \%$ do comprimento do intestino delgado. Entretanto, é sede de metade a $2 / 3$ dos adenocarcinomas desta víscera, o que pode ser explicado pela grande variedade de agentes potencialmente agressores que têm contato com a mucosa duodenal, como as secreções biliar, pancreática e gástrica ${ }^{5}$.

Embora descrito pela primeira vez em 1746 por Hamburg ${ }^{6}$, a raridade desse tipo de câncer ainda não nos permite identificar sua etiologia ${ }^{2}$. No entanto, doentes com polipose familial adenomatosa (síndromes de Gardnere Turcot), síndromes de Lynch e de Von Recklinghausen, doenças de Crohn e celíaca têm maior propensão para desenvolver tumor duodenals.

\footnotetext{
*Correspondência:

Rua Dr. Cesário Motta Jr., 112

01221-020 - S. Paulo - SP
}

Os sintomas e sinais mais comuns são os relacionados à obstrução intestinal, emagrecimento e anemia provocada por hemorragia digestiva crônica ${ }^{2,7,8}$. Os exames radiológicose endoscópicos sugerem o diagnóstico que é confirmado pelo exame anatomopatológico ${ }^{8}$.

0 tratamento adequado é o cirúrgico. Para os tumores localizados na segunda porção duodenal, a duodenopancreatectomia com remoção dos linfonodos regionais é o procedimento que satisfaz os princípios técnicos da cirurgia oncológica curativa ${ }^{9,10}$ Para as lesões da primeira, terceira e quarta porções, a ressecção segmentar pode ser indicada em casos selecionados ${ }^{9,11}$.

Ainvasão parietal, a metástase linfonodal ${ }^{7,8}$ e a localização no duodeno ${ }^{9,12}$ são os fatores prognósticos mais importantes. As lesões supra e peri-ampulares têm prognóstico pior que as infra-ampulares $8,9,13,14$.

Os tratamentos quimioterápico e/ou radioterápico não têm beneficiado a sobrevivência dos doentes operados. Entretanto, as publicações a esse respeito são escassas ${ }^{7,13}$.

A taxa de sobrevivência em cinco anos dos doentes com tumor extirpado com intenção curativa é $50 \%$ ". A ressecção pancreática e duodenal está associada aos melhores índices prognósticos ${ }^{15}$.

Revisamos a literatura especializada a respeito dos adenocarcinomas duodenais primários e comparamos os dados com os aspectos observados nos seis doentes desta casuística.

\section{Métodos}

Acompanhamos seis doentes com adenocarcinoma primário de duodenonaÁreadeEstômago e Duodeno do Departamento de Cirurgia da Faculdade de Ciências Médicas da Santa Casa de São Paulo, no período de 199| a 1999. Excluímos aqueles com tumores periampulares. Cincoeram dosexomasculino. Amédia etáriafoi 59,6 anos, variando de 49 a 82 anos.

Avaliamos os sintomas e sinais, os exames diagnósticos pré-operatórios, a localização eo tamanho do tumor, o procedimento cirúrgico utilizado e a evolução pós-operatória.

Todos foram operados. Três tiveram ressecções consideradas curativas e outros três, operações paliativas.

O seguimento pós-operatório, nos doentes com ressecção curativa, pretende o diagnóstico de recidivas e, naqueles com operações paliativas, o controle dos sintomas. 


\section{Resultados}

Todos os doentes procuraram atendimento médico com sintomas há pelo menos seis meses. Os mais comuns foram dor abdominal e emagrecimento (83,3\%), empachamento evômitos pós-prandiais (66,6\%), anemia e tumor palpável (16,6\%).

Empregaram-se a endoscopia digestiva alta e a ultra-sonografia em todos os doentes como métodos diagnósticos. Entretanto, 0 exame ultra-sonográfico, bem como a tomografia computadorizada utilizada em três deles, ajudou somente na definição do estádio da doença. Em um doente, iniciamos a investigação diagnóstica pela duodenografia, que revelou lesão tumoral na primeira porção duodenal. O exame endoscópico e a biópsia confirmaram a neoplasia. Noutro, com tumor localizado na terceira porção duodenal, havia anemia, provocada por hemorragia digestiva crônica ao longo de um ano (recebeu 36 unidades de concentrado de hemácias neste intervalo). Neste período, foram realizadas duas esofagogastroduodenoscopias, que não evidenciaram a doença, colonoscopia e angiografias do tronco celíaco, artéria mesentérica superior e inferior, que se mostraram normais, na tentativa de identificar o ponto de sangramento. Realizou-se, posteriormente, cintilografia com hemácias marcadas com tecnécio, que revelou hiperconcentração do fármaco no hipocôndrio direito e mesogástrio. Nova esofagogastrosduodenoscopia, desta vez, localizou o tumor.

O local mais freqüente dos tumores foi a primeira porção duodenal (quatro doentes). Além desses, tivemos um na segunda e outro na terceira porção. $O$ tamanho dos tumores variou de $2 \mathrm{a} 20 \mathrm{~cm}$ de dimensão máxima (média de $10,3 \mathrm{~cm}$ ). O exame histopatológico revelou adenocarcinoma bem diferenciado em dois e moderadamente diferenciado nos outros quatro.

Utilizamos a laparotomia em todos os doentes. Em três, executaram-se ressecções segmentares duodenais curativas. Em um, o tumor estava confinado à mucosa duodenal, em outro na submucosa e no terceiro invadia a serosa, sem ultrapassá-la. Os demais com a neoplasia invadindo órgãos adjacentes sofreram desvio do trânsito duodenal (gastrojejunostomia paliativa). Um deles foi reoperado por evisceração abdominal seis dias depois, e faleceu no $15^{\circ}$ dia de pós-operatório por complicações sépticas (mortalidade operatória de 16,6\%). O doente que apresentava adenocarcinoma da segunda porção duodenal necessitou também de derivação biliar (colecistojejunostomia).

O tempo de internação pós-operatória variou de 5 a 9 dias, com média de 7,3 dias. Em nenhum doente indicamos tratamento complementar com quimio ou radioterapia.

$O$ acompanhamento ambulatorial vem ocorrendo em períodos que variam de 15 a 20 meses. Os três pacientes submetidos à ressecção cirúrgica curativa estão sem evidências de recidiva até $o 15^{\circ}, 16^{\circ}$ e $18^{\circ}$ mês de pós-operatório. Houve seguimento de um e três meses nos dois pacientes submetidos a cirurgias paliativas, depois do que se perderam.

\section{Discussão}

Os adenocarcinomas são os neoplasmas malignos mais freqüentes no duodeno, seguidos pelos tumores carcinóides, leiomiossarcomas e linfomas ${ }^{5,13,16}$. Vários autores $2,5,9,14$ demonstraram que 0 tumor duodenal predomina no sexo masculino entre a $5^{\mathrm{a}}$ e a $7^{\mathrm{a}}$ décadas de vida, como constatamos em nossos doentes.

O quadro clínico é inespecífico e de início insidioso, podendo ser interpretado como decorrente de condições benignas do sistema digestório e tratados pelos próprios pacientes ou pelos médicos sem investigação detalha$\mathrm{da}^{7,9,10,16}$. Os sinais e sintomas mais freqüentes estão relacionados à sub-oclusão intestinal crônica, tais como dor abdominal relatada por $31,8 \%$ a $56 \%$, empachamento e vômitos pósprandiais referidos por $27,3 \%$ a $51,8 \%$, emagrecimento citado em $10,6 \%$ a $62 \%$ das vezes e anemia constatada em $27,3 \%$ a $60,6 \%$ dos doentes ${ }^{6,8,14}$. Em nossa casuística, o emagrecimento foi a queixa mais comum $(83,3 \%$ ) seguido daquelas relacionadas à sub-oclusão intestinal (66,6\%). Os sintomas duraram seis meses, em média, como registrado na literatura ${ }^{7,9,14}$.

O diagnóstico precoce é incomum e fortuito em exames de imagem ou durante ato operatório, por não haver quadro clínico característico ${ }^{8,14}$. Entretanto, com exames radiológicose endoscópicos minuciosos, o diagnóstico desta doença não é difícil ${ }^{7}$. Atualmente, com o desenvolvimento da fibroscopia, a pos- sibilidade de sua detecção em fase inicial tornou-se possível ${ }^{5}$. A gastroduodenoscopia e a duodenografia são os principais meios diagnósticos ${ }^{8}$. A ultra-sonografia e a tomografia computadorizada têm importância para definir o estádio pré-operatório da doença ${ }^{14,16}$. A arteriografia seletiva do tronco celíaco e da mesentérica superior permite avaliar a extirpabilidade da lesão quando há suspeita de invasão vascular pelo tumor ${ }^{14}$. Realizamos esse exame em um doente para diagnosticar o sítio de sangramento, sem êxito. Quando a icterícia se faz presente, está indicada a colangiografia retrógrada endoscópica ${ }^{14}$. Em nosso estudo, a gastroduodenoscopia fez o diagnóstico em todos os doentes. No caso do tumor localizado na terceira porção duodenal, somente o terceiro exame endoscópico revelou a lesão, pois a avaliação daquela porção da víscera não é habitual durante o procedimento. O neoplasma foi alcançado pelo aparelho, devido ao conhecimento, pela cintilografia com hemácias marcadas, da existência de sangramento na região do hipocôndrio direito e mesogástrio. Esta observação enfatiza a necessidade de realização de exame endoscópico meticuloso em doentes com queixas de sub-oclusão ou obstrução crônica alta do tubo digestório, pela possibilidade de lesões duodenais infraampulares.

A neoplasia duodenal é usualmente classificada de acordo com sua posição relativa à ampola de Vater. As lesões localizadas entre o piloro e essa estrutura são denominadas supra-ampulares. Os tumores que surgem na mucosa adjacente à ampola são definidos como peri-ampulares, localização mais freqüente. Aqueles que se encontram abaixo, até o ligamento de Treitz, são os infraampulares ${ }^{6}$. Na nossa casuística, quatro doentes apresentavam tumor na primeira porção $(66,6 \%)$, um na segunda $(16,6 \%)$ e outro na terceira porção duodenal (16,6\%). $\mathrm{Na}$ literatura consultada, parece não haver local preferencial. Encontramos relatos sobre incidência de tumores na primeira porção duodenal em $7 \%$ a $40 \%$, na segunda em $7 \%$ a $81,8 \%$, e na terceira e quarta porções entre $9,1 \%$ e $56,3 \%$ dos doentes $3,4,6$.

0 tratamento apropriado para o adenocarcinoma do duodeno é a extirpação cirúrgica, se possível, ou desvio do trânsito quando $\circ$ tumor não pode ser removi- 
do ${ }^{3,4,6,7,7,11,14}$. Para as lesões neoplásicas localizadas na segunda porção duodenal, a duodenopancreatectomia com linfonodectomia é o tratamento de escolha ${ }^{8,9}$. A ressecção segmentar é indicada para os tumores da terceira e quarta porções duodenais, com excelente taxa de sobrevivência e baixa morbidade pós-operatória $3,7,8,9,10,11$. A duodenopancreatectomia tem morbidez maior que a ressecção segmen$\operatorname{tar}$ duodenal| ${ }^{10,13}$. Na nossa casuística, três doentes foram submetidos à ressecção segmentar duodenal curativa, dois dos quais tinham tumores na primeira porção duodenal e outro na terceira. Nos que apresentavam neoplasia invadindo órgãos adjacentes, realizamos desvio do trânsito duodenal (gastrojejunostomia paliativa). No único caso de adenocarcinoma de segunda porção duodenal, fizemos, além do desvio do trânsito do duodeno, a derivação biliar (colecistojejunostomia). A resseçcão endoscópica pode ser tentada em lesões precoces, desde que não tenham depressão central ou metástase linfonodal ${ }^{17}$.

De acordo com alguns autores ${ }^{6,8,9}$, a localização da neoplasia, a invasão tumoral parietal e a metástase linfonodal são os fatores prognósticos mais importantes. Em nosso estudo, os carcinomas invadiam órgãos adjacentes (T4) em três doentes; em um, atingia a serosa, sem ultrapassá-la (T3); em outro, alcançava a submucosa $(\mathrm{TI})$ e no último, estava restrito à $\operatorname{mucosa}(\mathrm{TI})$.

Alinfonodectomia é significativa para o aumento da taxa de sobrevivência ${ }^{8,14}$. No doente que apresentava neoplasia precoce (restrito à mucosa duodenal), extirparam-se oito linfonodos, nenhum com invasão tumoral (NO). Naquele cujo tumor invadia a submucosa, retiraram-se I 3 linfonodos, um deles positivo(NI). No doente em que o tumor infiltrava a serosa, não foi encontrado qualquer linfonodo na peça cirúrgica. Nossos doentes não desenvolveram metástases durante o curto período de seguimento. Aletalidade operatória, definida como 0 óbito ocorrido desde a operação até 30 dias da alta hospitalar, ocorreu em $0 \%$ a $25 \%$ dos casos da literatura consultada ${ }^{3,4}$. As principais causas de morte foram as complicações sépticas em $50 \%$ das vezes e as cardiopulmonares na outra metade ${ }^{4}$. A taxa de sobrevivência em cinco anos foi de $15 \%$ a $38 \% \%^{2,4,8}$. Entretanto, em tumores comoperação curativa, essa taxa elevou-se para $28 \%$ a $86 \%$ 2,4. Tivemos apenas um óbito $(16,6 \%)$ em doente com tumor avançado submetido a procedimento paliativo. Vários estudos têm demonstrado que a quimioterapia e a radioterapia não beneficiam, de forma significativa, a sobrevivência dos pacientes operados ${ }^{7,12}$, talvez porque o pequeno número de casos relatados não permitiu ainda estudos mais consistentes.

\section{SUMMARY}

AdeNocarcinoma of the dUODENUM

PURPOSE. To revise literature data about adenocarcinoma of the duodenum comparing to ourresults.

MetHOds. Six patients with this kind of cancer, excluding periampullarylesions, were treated from 1991 to 1999 in the Department of Surgery, Santa Casa ofSão Paulo MedicalSchool. Five of them were males in theirs fifties. The commonest signs and symptoms were those of gastrointestinal obstruction and weight loss. All patients were evaluated by duodenoscopy. Ultrasonography and CT scans played an important role with regard to preoperative staging. Three patients underwent a segmental resection and the others were submitted to a palliative duodenal bypass.

RESULTS. Post-operative hospitalization varied from 5 to 9 days (7,3 days average). The operative mortality was $16.6 \%$ (one patient). Patients were not submitted to chemo or radiotherapy. Follow-up has been done. Patients with curative resection were free from recurrence. Two patients submitted to palliative operationswerelost to follow-up.

CONCLUSION. Rarity of duodenum carcinomas and few patients in the literature did not permit conclusions. [Rev Assoc Med Bras 2002; 48(3): 242-4].

KEY WORDS: Primary adenocarcinoma. Duodenum.

\section{RefERÊNCIAS}

I. Souza HP, Toneto MG, Bonfati MG, Pandolfo G. Adenocarcinoma primário de duodeno. Rev Col Bras Cir 1999; 26: I 22-4.

2. Cortese AF, Cornell GN. Carcinoma of the duodenum. Cancer 1972; 29:1010-5.
3. Spira IA, Ghazi A, Wolff WI. Primary adenocarcinoma of the duodenum. Cancer 1977; 39:1721-6.

4. Alwmark A, Anderson A, Lasson A. Primary carcinoma of the duodenum. Ann Surg 1980; 191:13-8.

5. Howe JR, Karnell LH, Menck RH, Scott-Conner C. Adenocarcinoma of the small bowel. Review of the National Cancer Data Base. I 985- 1995. Cancer 1999; 86:2693-706.

6. Scott-Coombs DM, Williamson RC. Surgical treatment of primary duodenal carcinoma: a personal series. BrJ Surg 1994; 81 : I 472-4.

7. Satake K, Sowa M, Yamashita K, Kamino K, Kinoshita $\mathrm{H}$, Kato M, et al. Carcinoma of the duodenum: Its preoperative diagnosis. $\mathrm{Br}$ J Surg 1975; 62:973-6

8. Heniford BT, lannitti DA, Evans P, Gagner M, Henderson JM. Primary nonampullary/periampullary adenocarcinoma of the duodenum. Am. Surg 1998; 64: I 165-9.

9. Joesting DR, Beart RW, Van Heerden JA, Weiland LH. Improving survival in adenocarcinoma of the duodenum. Am J Surg 198I; |41:228-31.

10. Delcore R, Thomas JH, Forster J, Hermreck AS. Improving resectability and survival in patients with primary duodenal carcinoma. Am J Surg 1993; 166:626-30.

II. Lowell JA, Rossi RL, Munson JL, Braasch JW. Primary adenocarcinoma of third and fourth portions of duodenum. Favorable prognosis after resection. Arch Surg 1992; 127:557-60.

12. Yildirim S, Culhaoglu AB, Ozdemir N. Carcinoma of the fourth part of the duodenum: report of a case. Jpn J Surg 1995; 25: 1034-7.

13. Lillemoe K, Imbembo AL. Malignant neoplasms of the duodenum. Surg Gynecol Obstet 1980; 150:822-6.

14. Santoro E, Sacchi M, Sartari F, Carboni F, Graziano F. Primary adenocarcinoma of the duodenum: Treatment and survival in 89 patients. Hepatogastroenterology 1997; 44:II57-63.

15. Bromberg SH, Goffi Jr PS, Goffi FS, Yamaguchi $\mathrm{N}$, Ussami E, Tolosa EM. Neoplasias duodenais primárias: análise de casuística e conduta cirúrgica. Rev Col Bras Cir 2000; 27:267-70.

16. Tanaka Y, Tokunaga K, Katsuragawa K, Yoshiura K, Naitoh M, Kino K, et al. Early carcinoma of the distal second part of the duodenum treated by wedge resection. J Clin Gastroenterol 1999;28:67-9

17. Hirasawa R, lishi $H$, Tatsuta M, Ishiguro S. Clinicopathologic features andendoscopic resection of duodenal adenocarcinomas and adenomas with the submucosal saline injection technique. Gastrointest Endosc 1997; 46:507-I 3.

Artigo recebido: 24/07/200I

Aceito para publicação: 23/08/200I 